\title{
Indirect Robust Estimation of the Short-term Interest Rate Process
}

\author{
Veronika Czellar ${ }^{1}$ \\ Dept. of Econometrics, University of Geneva, Switzerland \\ G. Andrew Karolyi \\ Fisher College of Business, Ohio State University \\ Elvezio Ronchetti \\ Dept. of Econometrics, University of Geneva, Switzerland
}

September 23, 2006

\begin{abstract}
We propose Indirect Robust Generalized Method of Moments (IRGMM), a simulationbased estimation methodology, to model short-term interest rate processes. The primary advantage of IRGMM relative to classical estimators of the continuous-time short-rate diffusion processes is that it corrects both the errors due to discretization and the errors due to model misspecification. We apply this approach to monthly US risk free rates and to various monthly Eurocurrency rates and provide extensive evidence of its predictive performances in a variety of settings.
\end{abstract}

Keywords: GMM and RGMM estimators, indirect inference.

JEL Classifications: G10, G12, C10, C22, C15, C53.

\footnotetext{
${ }^{1}$ Much of this work was completed while V. Czellar was visiting the Departement of Finance of the Fisher College of Business at Ohio State University whose hospitality is gratefully acknowleged. She would like to thank also Lombard Odier Darier Hentsch\&Cie (Geneva) for the financial support of this visit. Also helpful comments by R. Stulz, J. Detemple, F. Trojani, seminar participants of BusFin 923 at the Fisher College of Business (Ohio State University), and participants at research seminars at Ohio State University, at University of Geneva, at University of Manchester and at Boston University are gratefully acknowledged. G. A. Karolyi thanks the Dice Center for Research in Financial Economics for financial support.
} 


\section{Introduction}

Understanding the dynamics of the short-term interest rate is important for building models of the term structure of interest rates, which, in turn, are used for pricing interest rate derivatives and instruments with embedded options such as callable bonds and mortgage-backed securities. Although there has been extensive analysis of the time series of short-term interest rates, some basic modeling issues remain unresolved. One issue stems from the difficulties associated with the statistical analysis of continuous-time processes. For example, in complex statistical models, like diffusion models described by stochastic differential equations (SDE) of the form,

$$
d y_{t}=\eta\left(y_{t}, \theta\right) d t+\sigma\left(y_{t}, \theta\right) d W_{t}
$$

where $\theta \in \mathbb{R}^{p}, \eta(\cdot)$ and $\sigma(\cdot)$ are the drift and the volatility, respectively, and $W_{t}$ a Wiener process, it is often difficult or impossible to carry out standard likelihood based estimation and inference. Such SDEs play an important role when modeling the short-term interest rate; see, for example, Vasicek (1977), Dothan (1978), Brennan and Schwartz (1977), Cox, Ingersoll and Ross (1981, 1985), Chan, Karolyi, Longstaff and Sanders (CKLS, 1992), Brenner, Harjes and Kroner (1996), Ahn and Gao (1999), among others.

A great deal of progress has been made recently in developing efficient tools for estimating and testing continuous-time models of the short-rate process. Important contributions include, for instance, the Efficient Method of Moments (Gallant and Tauchen, 1996; Andersen and Lund, 1997), weighted least squares estimation (Chapman and Pearson, 1999), simulated maximum likelihood estimation (Durham and Gallant, 2002; Durham, 2003), Gibbs sampling-based Markov Chain Monte Carlo algorithms (Elerian, Chib and Shephard, 2001; Eraker, 2001), and retrospective sampling-based Monte Carlo methods (Beskos, Papaspiliopoulos, Roberts and Fearnhead, 2006).

It is well known that estimators based on a discretized version of (1) are biased, see for instance Gouriéroux, Monfort and Renault (1993). To tackle the problem arising from discretization, several approaches have been proposed. Among others, non-parametric techniques (Aït-Sahalia, 1996; Stanton, 1997; Pritsker, 1998; Hong and Li, 2005; Johannes, 2004), pseudo-likelihood estimation (Aït-Sahalia, 1999; Aït-Sahalia, 2002) and indirect techniques (Broze, Scaillet and Zakoïan, 1995) have been used to estimate the short-term interest rate process. An additional problem that arises when estimating the short rate process is the possible misspecification of the model which can lead to biased estimators and misleading test results. The theory of robust statistics can be used to mitigate this problem. Specifically, Dell'Aquila, Ronchetti and Trojani (2003) used the Robust Generalized Method of Moments (RGMM) technique, developed by Ronchetti and Trojani (2001), to estimate discrete-time interest rate processes.

Typically the estimation of (1) is performed by means of an auxiliary model which is a discretized version of the SDE. The resulting indirect estimation (Gouriéroux et al., 1993) is based on the following idea. Given a sample of observations $\left\{y_{t}\right\}_{t=1, \ldots, n}$ assumed to be generated from a probability model $F_{\theta}, \theta \in \mathbb{R}^{p}$, define an auxiliary model $\tilde{F}_{\mu}$ where the parameter $\mu \in \mathbb{R}^{r}$ is easier to estimate than $\theta$. For instance, $F_{\theta}$ could be the diffusion 
model (1) or a fine discretization of (1) and $\tilde{F}_{\mu}$ a crude discretization of (1). The main steps of indirect estimation are:

(i) an auxiliary estimator $\tilde{\mu}$ is calculated with the original sample;

(ii) pseudo-observations are simulated from the model $F_{\theta}$ generating $S$ samples of pseudo-data

$$
\left\{y_{t}^{1}(\theta)\right\}_{t=1, \ldots, n}, \ldots,\left\{y_{t}^{S}(\theta)\right\}_{t=1, \ldots, n}
$$

(iii) an auxiliary estimator $\tilde{\mu}_{S}(\theta)$ is calculated with the simulated pseudoobservations;

(iv) the indirect estimator $\hat{\theta}$ of $\theta$ is obtained as that which minimizes the distance between the auxiliary estimators $\tilde{\mu}$ and $\tilde{\mu}_{S}(\theta)$ :

$$
\hat{\theta}=\arg \min _{\theta}\left(\tilde{\mu}-\tilde{\mu}_{S}(\theta)\right)^{T} \Omega\left(\tilde{\mu}-\tilde{\mu}_{S}(\theta)\right) .
$$

Gouriéroux and Monfort (1996) proved that, under certain regularity conditions, the indirect estimator $\hat{\theta}$ is consistent for $\theta$ and asymptotically normal. Moreover, if the bias of the auxiliary estimator is of order $\mathcal{O}(1)$, then indirect estimation reduces this bias. That is, the bias of $\hat{\theta}$ is of order $\mathcal{O}\left(n^{-1}\right)$.

This procedure relies on the assumption that the underlying model $F_{\theta}$ is exact, or, in other words, that it has generated the data. If the underlying model $F_{\theta}$ is misspecified and the real data is generated from a perturbed model such as $(1-\varepsilon) F_{\theta}+\varepsilon G$, where $G$ is an unknown distribution and $0 \leq \varepsilon<1$, Genton and Ronchetti (2003) showed that even the indirect estimators are biased. To eliminate this bias, they developed robust indirect estimation which is based on a robust estimator of the auxiliary parameter. They proved that, if the auxiliary estimator is a consistent and robust estimator of the parameter, then the associated indirect estimator is also robust. In the same spirit, Ortelli and Trojani (2005) extended these results to the broader setup of the efficient method of moments and developed robust techniques in this case by providing efficient algorithms for their computation.

In this paper, we combine these results and apply them to a statistical analysis of models described by (1). In particular, we define the indirect robust Generalized Method of Moments (IRGMM), a simulation-based estimation of SDEs which reduces both the bias due to discretization and to contamination, and apply it to monthly US risk free and to various monthly Eurocurrency interest rate series. We also investigate three different diffusion models for short-term interest rates proposed in the finance literature (CKLS, 1992; Ahn and Gao, 1999; Chapman and Pearson, 2000) to explore the sensitivity of our findings to different model specifications. We compare empirical results obtained with GMM, RGMM and IRGMM in both within- and out-of-sample predictive tests. For many of the cases, we obtain superior predictive performances using the IRGMM estimation technique.

The paper is organized as follows. In Section 2, we introduce the IRGMM technique to estimate short-term interest rate processes and explain the advantages of this procedure 
with respect to classical estimation methods. Section 3 presents the results of IRGMM estimation of monthly US one-month risk free rates with three different diffusion models and three different techniques: GMM, RGMM and IRGMM. We compare their performances by means of within-sample goodness-of-fit tests. In Section 4, we compare the techniques and models presented in Section 3 using various monthly Eurocurrency rates. We compute out-of-sample goodness-of-fit tests and evaluate the predictive performance of the IRGMM estimation-based forecasts in the period from 2000-2004. Finally, Section 5 concludes the article with some suggestions for further work.

\section{IRGMM analysis of the short-term interest rate process}

\subsection{Robust indirect inference}

A necessary condition for the consistency of an indirect estimator defined in (2) is that the original data $\left\{y_{t}\right\}$ and the pseudo-data are generated from the same probability model, see Gouriéroux et al. (1993). In reality, the presence of large outliers and high kurtosis in the increments of interest rate data shows that a diffusion model with standard normal increments may be misspecified. As an illustration, let us consider the diffusion model (1) where the increments come from an $\varepsilon$-neighborhood of a standard normal distribution defined by:

$$
W_{t}-W_{t-1}=\epsilon_{t} \sim G_{\varepsilon}=(1-\varepsilon) \mathcal{N}(0,1)+\varepsilon G,
$$

with $0 \leq \varepsilon<1$ and $G$ an unknown distribution.

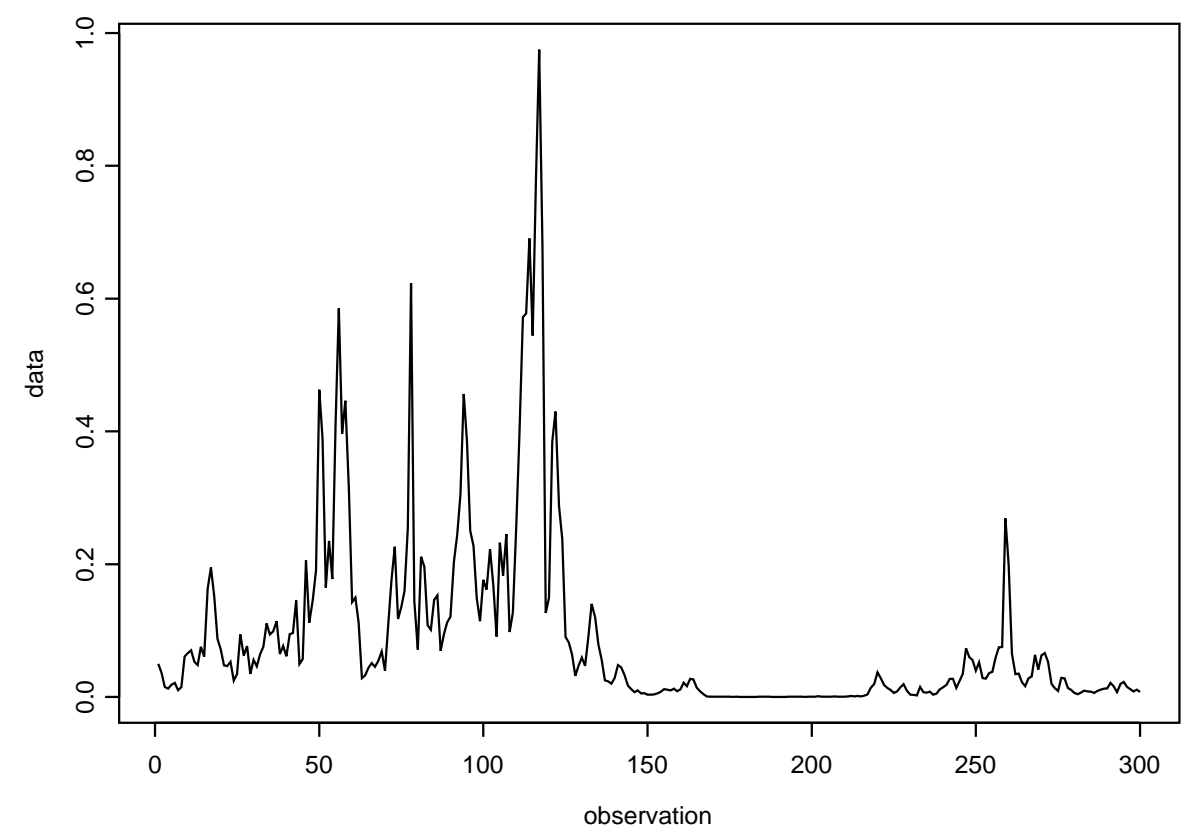

Figure 2.1: Simulated data from a GBM diffusion model with $5 \%$ contamination. 
As an illustrative example, Figure 2.1 presents simulated data of sample size $n=300$ generated from a contaminated geometric Brownian motion with drift ${ }^{2}$ :

$$
d y_{t}=\beta y_{t} d t+\sigma y_{t} d W_{t},
$$

where the increments follow $G_{\varepsilon}=(1-\varepsilon) \mathcal{N}(0,1)+\varepsilon t_{3}$ with $\varepsilon=0.05, \beta=0.1, \sigma=0.5$ and $t_{3}$ a $t$-distribution with 3 degrees of freedom. Figure 2.1 shows that a contaminated diffusion model can generate large outliers in the data. The kurtosis of $y_{t}-y_{t-1}$ for the plotted sample is approximately 10.50, which is much higher than 3 , the kurtosis under the normal model.

Our goal is to construct an indirect estimator of the diffusion model (1) which is robust to misspecifications of the underlying stochastic structure of the model described in (3). Genton and Ronchetti (2003) have shown that indirect estimators are robust if the auxiliary estimator is a robust estimator of the parameter of the auxiliary model. In our case, the auxiliary model $\tilde{F}_{\mu}$ is a discretization of the diffusion model (1):

$$
y_{t}=y_{t-1}+\eta\left(y_{t-1}, \mu\right)+\sigma\left(y_{t-1}, \mu\right) \epsilon_{t},
$$

where $\left\{\epsilon_{t}\right\}_{t=1, \ldots, n}$ are independent, identically-distributed standard normal variables. Hereafter we will refer to equation (5) as the crude discretization. In order to use Genton and Ronchetti's result to construct a robust indirect estimator of the parameter $\theta$ of the diffusion model, we need a robust estimator of the parameter $\mu$. The robust version of the GMM estimator (see Ronchetti and Trojani, 2001) is a GMM estimator where the classical $H$-dimensional orthogonality condition $h$ is replaced by a truncated orthogonality condition:

$$
h_{c}^{A, \tau}\left(y_{t}, y_{t-1}, \mu\right)=A\left[h\left(y_{t}, y_{t-1}, \mu\right)-\tau\right] w_{c}\left(y_{t}, y_{t-1}, \mu\right),
$$

where $c>\sqrt{H}$ is a tuning constant and $w_{c}\left(y_{t}, y_{t-1}, \mu\right)=\min \left(1, \frac{c}{\left\|A\left[h\left(y_{t}, y_{t-1}, \mu\right)-\tau\right]\right\|}\right)$ is a weight assigned to $y_{t}$ which is smaller than 1 when $\left\|A\left[h\left(y_{t}, y_{t-1}, \mu\right)-\tau\right]\right\|>c$. The nonsingular matrix $A \in \mathbb{R}^{H} \times \mathbb{R}^{H}$ and the vector $\tau \in \mathbb{R}^{H}$ are determined by the implicit equations

$$
\begin{gathered}
E_{\mu_{0}} h_{c}^{A, \tau}=0, \\
\frac{1}{n} \sum_{t=1}^{n} h_{c}^{A, \tau}\left(h_{c}^{A, \tau}\right)^{T}=I .
\end{gathered}
$$

where for simplicity $h_{c}^{A, \tau}=h_{c}^{A, \tau}\left(y_{t}, y_{t-1}, \mu\left(\tilde{F}_{\mu_{0}}\right)\right)$. An iterative algorithm for the computation of an RGMM estimator is defined in Ronchetti and Trojani (2001, p.47).

Denote by $\tilde{\mu}$ the robust GMM (RGMM) estimator associated with the truncated orthogonality function (6). By construction, the RGMM estimator is defined by:

$$
\begin{gathered}
\tilde{\mu}=\arg \min _{\mu} g\left(\left\{y_{t}\right\}, \mu\right) \\
g\left(\left\{y_{t}\right\}, \mu\right)=\frac{1}{n} \sum_{t=1}^{n} h_{c}^{A, \tau}\left(y_{t}, y_{t-1}, \mu\right)^{T} \frac{1}{n} \sum_{t=1}^{n} h_{c}^{A, \tau}\left(y_{t}, y_{t-1}, \mu\right) .
\end{gathered}
$$

\footnotetext{
${ }^{2}$ This case has an "exact discretization": $\log \left(\frac{y_{t}}{y_{t-1}}\right)=\beta-\frac{\sigma^{2}}{2}+\sigma \epsilon_{t}$ with $\epsilon_{t} \sim G_{\varepsilon}$.
} 
We define the indirect robust GMM (IRGMM) estimator as an indirect estimator computed with an auxiliary RGMM estimator. In Section 2.2 we present an algorithm to compute the IRGMM estimator.

\subsection{The IRGMM technique}

We define the IRGMM estimator $\hat{\theta}$ according to the following steps:

1) Compute the constant matrix $A$, vector $\tau$, and the RGMM estimator $\tilde{\mu}$ defined in (7)-(10) using the real data $\left\{y_{t}\right\}_{t=0,1, \ldots, n}$.

2) Simulate pseudo-observations from a fine discretization of (1) by dividing the time interval $\Delta t=1$ into $m=1 / \delta$ subintervals of length $\delta$. The Euler approximation corresponding to the time interval $\delta$ is the process $\left\{y_{k \delta}\right\}_{k=0,1, \ldots, m n}$ defined by

$$
y_{(k+1) \delta}=y_{k \delta}+\delta \eta\left(y_{k \delta}, \theta\right)+\sqrt{\delta} \sigma\left(y_{k \delta}, \theta\right) \epsilon_{k},
$$

where $\epsilon_{k}$ is a standard normal variable. Selecting data at times $k \delta \in \mathbb{N}$, obtain pseudo-data $\left\{y_{t}^{(s)}(\theta)\right\}_{t=0,1, \ldots, n}$. Simulate $S$ pseudo-data sets from this model:

$$
\left\{y_{t}^{s}(\theta)\right\}_{t=0,1, \ldots, n}, \quad s=1, \ldots, S, \quad S \geq 1 .
$$

3) For each $s$, calculate the function $g\left(\left\{y_{t}^{s}(\theta), \mu\right)\right.$ defined in (10) using $A$ and $\tau$ obtained in 1) and the auxiliary estimator for the pseudo-data:

$$
\tilde{\mu}_{S}(\theta)=\arg \min _{\mu} \sum_{s} g\left(\left\{y_{t}^{s}(\theta)\right\}, \mu\right) .
$$

4) The IRGMM estimator $\hat{\theta}$ is that which minimizes the distance between the auxiliary estimators $\tilde{\mu}$ and $\tilde{\mu}_{S}(\theta)$ :

$$
\hat{\theta}=\arg \min _{\theta}\left(\tilde{\mu}-\tilde{\mu}_{S}(\theta)\right)^{T} \Omega\left(\tilde{\mu}-\tilde{\mu}_{S}(\theta)\right),
$$

where $\Omega$ is a positive definite symmetric matrix, for instance, the inverse of the asymptotic covariance matrix of the auxiliary estimator.

Notice that the computational complexity of this algorithm can be reduced by using techniques developed in Ortelli and Trojani (2005). The IRGMM estimation procedure is summarized in Figure 2.2. Since the RGMM estimator is a robust estimator of the auxiliary parameter $\mu$, the IRGMM is a consistent and robust estimator of the diffusion parameter $\theta$ (for consistency of indirect estimators see Gouriéroux et al., 1993; for robustness of indirect estimators see Genton and Ronchetti, 2003). The IRGMM estimator corrects both the errors due to discretization and the errors due to contamination of the underlying diffusion model. 


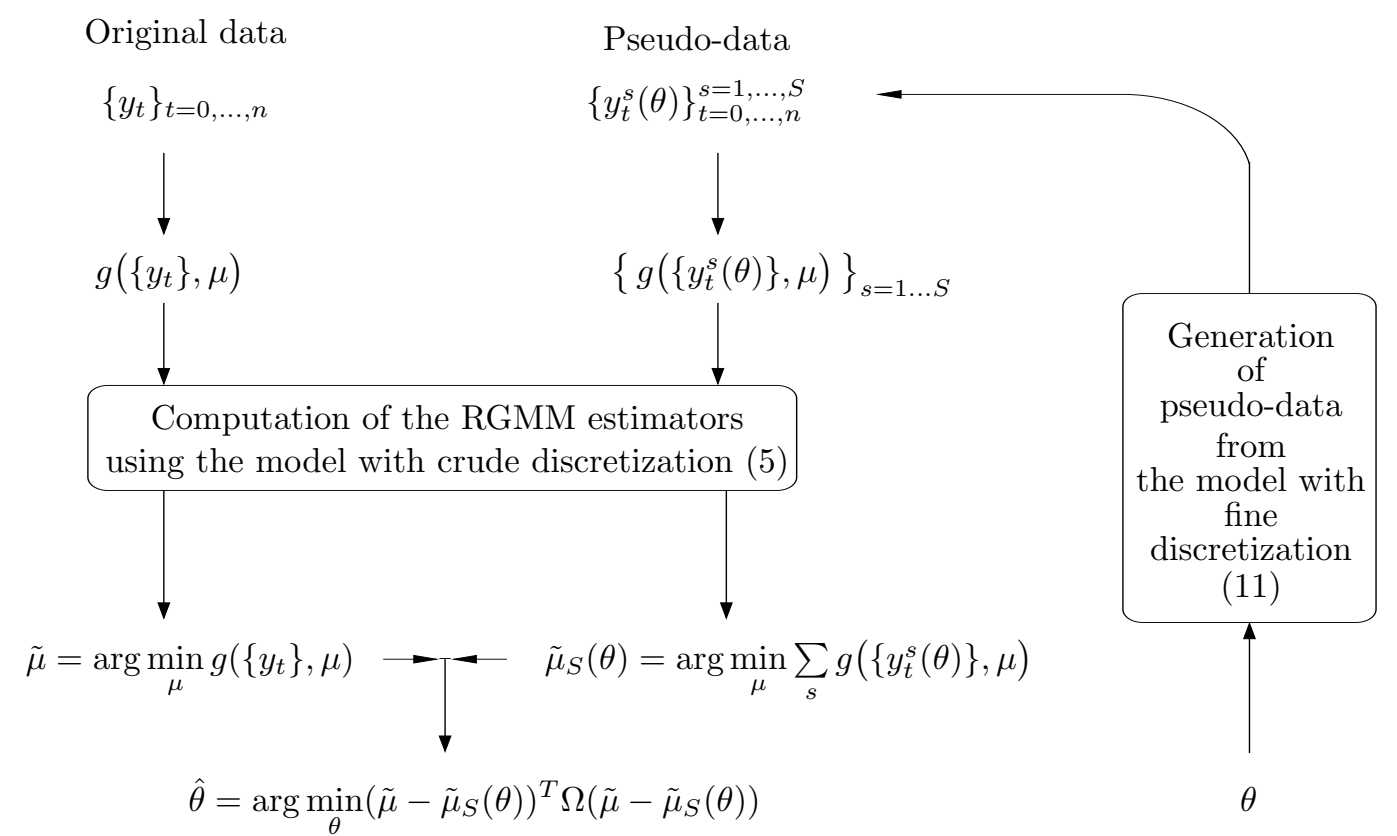

Figure 2.2 Construction of the IRGMM estimator $\hat{\theta}$

\subsection{The choice of the parameters $S$ and $\delta$}

To calibrate our procedure, we generate data of sample size $n=100$ from the exact discretization of (4) with $\beta=0.1, \sigma=0.5$ and standard normal increments. We computed indirect estimators with auxiliary maximum likelihood estimators of the crude discretization of $(4)^{3}$. In Figures 2.3-2.4 we plot the indirect parameter estimates of $\beta$ and $\sigma$ obtained with $S=10,100,1000$ pseudo-data sets and with increasing values of $1 / \delta$. The plots show that there is no significant gain in stability of the indirect estimator computed with $S=1000$ compared to the one computed with $S=100$ if we take into account the fact that computation time is increasing linearly with $S$.

Figure 2.4 shows that the indirect estimator settles down for $1 / \delta \geq 10$, so a choice of

\footnotetext{
${ }^{3}$ The crude discretization of (4) is

$$
y_{t}=y_{t-1}+\beta y_{t-1}+\sigma y_{t-1} \epsilon_{t}
$$

and the maximum likelihood estimators for $\beta$ and $\sigma^{2}$ are

$$
\tilde{\beta}=\bar{r}-1, \quad \tilde{\sigma}^{2}=\frac{1}{n} \sum_{t=1}^{n}\left(r_{t}-\bar{r}\right)^{2}
$$

with $r_{t}=y_{t} / y_{t-1}$ and $\bar{r}=1 / n \sum_{t=1}^{n} r_{t}$.
} 
$\delta \leq 1 / 10$ is necessary. In the next section, we estimate monthly rates and in this case it is natural to choose $\delta=1 / 22$ for the simulation of daily rates (given there are about 22 trading days per month). By around $1 / \delta=22$ for the case with $S=100$ replications, Figures 2.3-2.4 indicate reasonable convergence is reached for both parameters of interest.
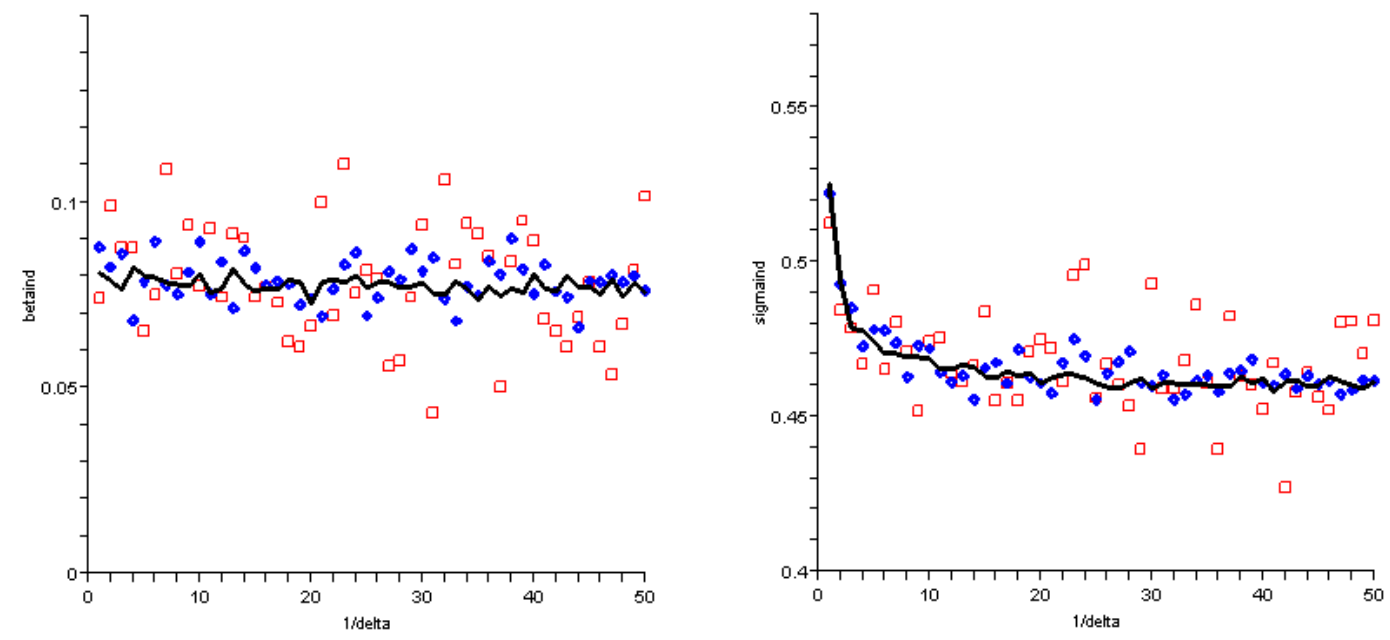

Figures 2.3-2.4 Indirect estimators of $\beta$ and $\sigma$ for $S=10$ (boxes), for $S=100$ (diamonds) and for $S=1000$ (curve).

\section{Model Estimation}

\subsection{The data}

We consider one month risk free rates from the Monthly CRSP Fama Risk Free Rates File covering the period from June 30,1964 to December 31,2004, which is an extension of the dataset used by Chan et al. (1992). The data is plotted in Figure 3.1 and Table 3.1 presents the means, standard deviations, skewness, kurtosis and autocorrelations of the monthly data.

\begin{tabular}{l|rrrrrrrrrr} 
Data & $N$ & Mean & StDev & Sk & $K u$ & $\rho_{1}$ & $\rho_{2}$ & $\rho_{3}$ & $\rho_{4}$ & $\rho_{5}$ \\
\hline \hline$y_{t}$ & 487 & 0.05667 & 0.02682 & 1.00730 & 1.78865 & 0.9652 & 0.9300 & 0.8993 & 0.8757 & 0.8558 \\
$\Delta y_{t}$ & 486 & -0.00003 & 0.00679 & -1.42971 & 14.79603 & 0.0082 & -0.0758 & -0.1133 & -0.0594 & -0.0139
\end{tabular}

Table 3.1: One month risk free rates statistics. 


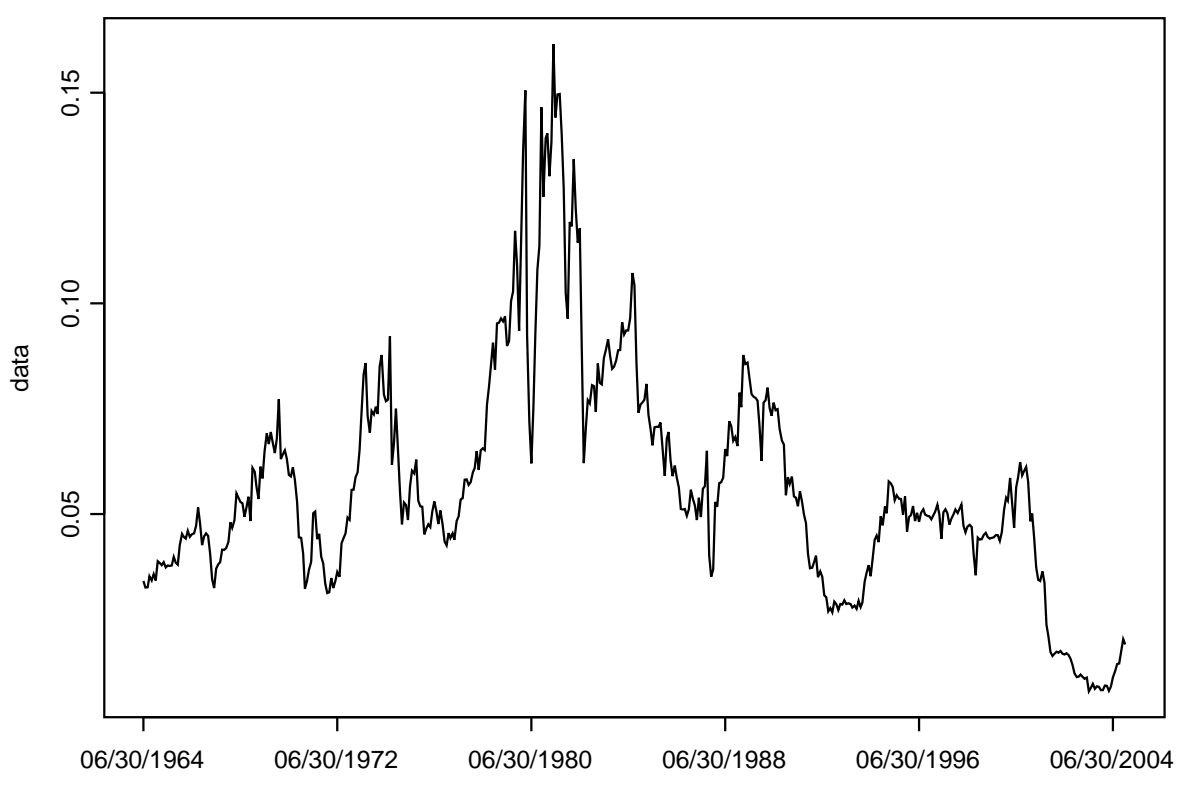

Figure 3.1: One month risk free rates from June 1964 to December 2004.

\subsection{The models}

Suppose the data is generated from a diffusion model (1) where $W_{t}$ is a Wiener process defined on monthly time intervals. Consider the following diffusion model:

$$
d y_{t}=\left(\beta_{0}+\beta_{1} y_{t}+\beta_{2} y_{t}^{2}+\frac{\beta_{3}}{y_{t}}\right) d t+\sigma y_{t}^{\gamma} d W_{t}
$$

This model has been investigated using nonparametric techniques by Aït-Sahalia (1996), using maximum likelihood by Aït-Sahalia (1999) and using GMM by Chapman and Pearson (2000). We also consider two alternative nested versions of (14): the CKLS model (Chan et al., 1992) and that of Ahn and Gao (1999). The restrictions for these alternative models are listed in Table 3.2.

\begin{tabular}{l|cccccc} 
& $\beta_{0}$ & $\beta_{1}$ & $\beta_{2}$ & $\beta_{3}$ & $\sigma$ & $\gamma$ \\
\hline \hline$C P$ & - & - & - & - & - & - \\
$C K L S$ & - & - & 0 & 0 & - & - \\
$A G$ & - & - & - & 0 & - & 1.5
\end{tabular}

Table 3.2: The models of Chapman and Pearson, CKLS and Ahn and Gao. 
We estimate our dataset using the three models and three different techniques: classical GMM, RGMM and IRGMM, as defined in Section 2. For the estimation of the unrestricted model by GMM, we consider Chapman and Pearson's (2000) orthogonality conditions:

$$
E\left[h\left(y_{t}, y_{t-1}, \mu\right)\right]=0, \quad h\left(y_{t}, y_{t-1}, \mu\right)=\left(\begin{array}{c}
\nu_{t} \\
\nu_{t} y_{t-1} \\
\nu_{t} y_{t-1}^{2} \\
\nu_{t} y_{t-1}^{-1} \\
\nu_{t}^{2}-\sigma^{2} y_{t-1}^{2 \gamma} \\
\left(\nu_{t}^{2}-\sigma^{2} y_{t-1}^{2 \gamma}\right) y_{t-1}
\end{array}\right)
$$

where $\nu_{t}=y_{t}-y_{t-1}-\beta_{0}-\beta_{1} y_{t-1}-\beta_{2} y_{t-1}^{2}-\beta_{3} / y_{t-1}$. For the CKLS model, we use the orthogonality conditions proposed by Chan et al., 1992):

$$
h\left(y_{t}, y_{t-1}, \mu\right)=\left(\begin{array}{c}
\nu_{t} \\
\nu_{t} y_{t-1} \\
\nu_{t}^{2}-\sigma^{2} y_{t-1}^{2 \gamma} \\
\left(\nu_{t}^{2}-\sigma^{2} y_{t-1}^{2 \gamma}\right) y_{t-1}
\end{array}\right),
$$

where $\nu_{t}=y_{t}-y_{t-1}-\beta_{1} y_{t-1}-\beta_{0}$. Finally for the GMM estimation of the Ahn and Gao's model, we use the orthogonality conditions proposed by Ahn and Gao (1999):

$$
h\left(y_{t}, y_{t-1}, \mu\right)=\left(\begin{array}{c}
\nu_{t} \\
\nu_{t} y_{t-1} \\
\nu_{t} y_{t-1}^{2} \\
\nu_{t}^{2}-\sigma^{2} y_{t-1}^{3} \\
\left(\nu_{t}^{2}-\sigma^{2} y_{t-1}^{3}\right) y_{t-1} \\
\left(\nu_{t}^{2}-\sigma^{2} y_{t-1}^{3}\right) y_{t-1}^{3}
\end{array}\right)
$$

where $\nu_{t}=y_{t}-y_{t-1}-\beta_{0}-\beta_{1} y_{t-1}-\beta_{2} y_{t-1}^{2}$. The orthogonality conditions defined in (15)(17) are also used for the determination of the truncated orthogonality conditions necessary for the computation of the RGMM and IRGMM estimators.

For the RGMM and IRGMM estimation, we set the tuning constant $c=5.85$ and the consistency parameter $\tau=0^{4}$. In this case the RGMM estimator used here is defined only by equations (8)-(10).

\subsection{Estimation and goodness-of-fit tests}

By construction, the RGMM estimation technique detects outliers and assigns weights to them between 0 and 1, see equation (6). Figures 3.3-3.5 show the weights of the observations attributed by the RGMM for each of the three models.

\footnotetext{
${ }^{4}$ The consistency parameter can be dropped since indirect inference corrects the inconsistency of the auxiliary estimator.
} 

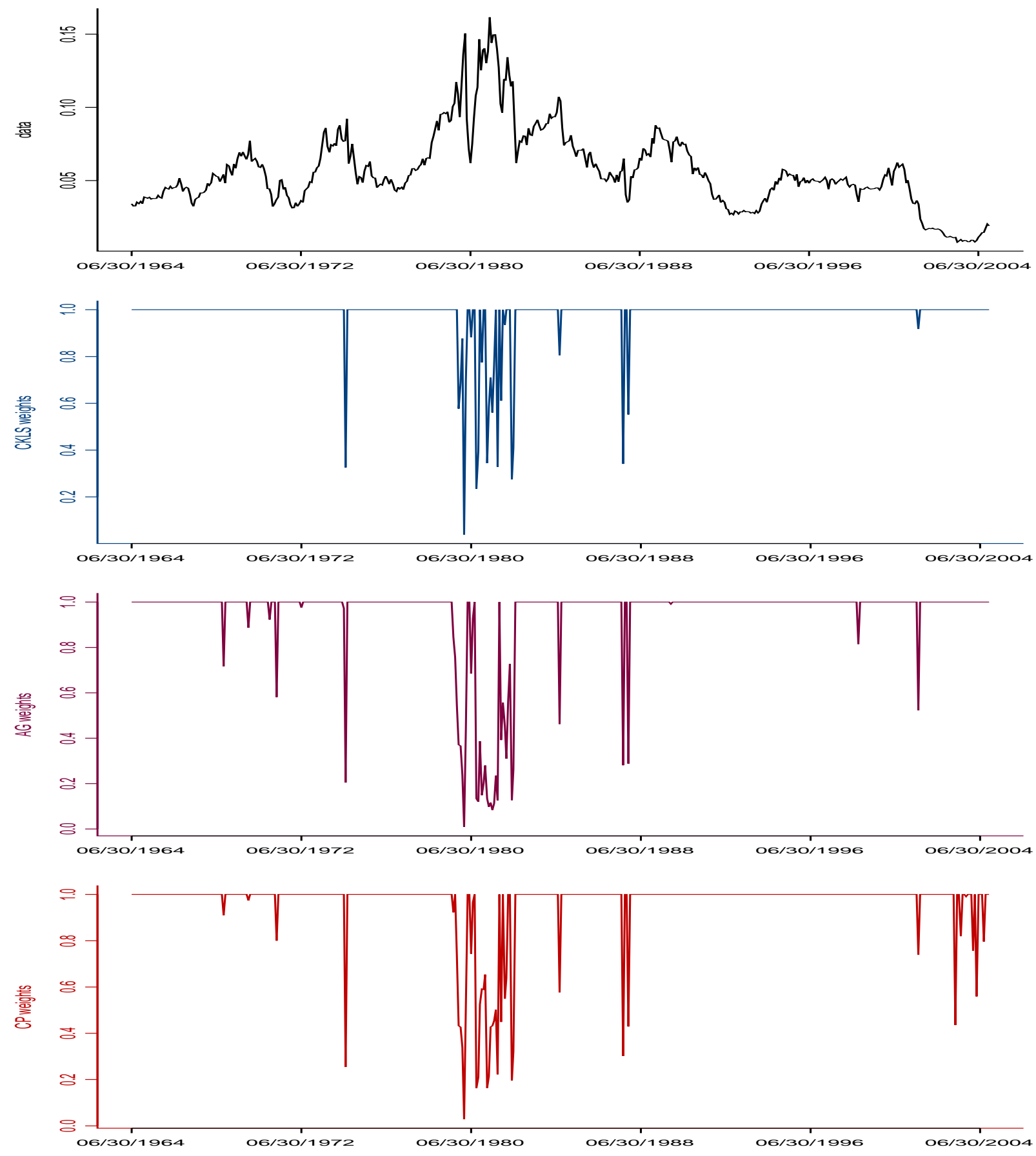

Figures 3.2-3.5: One month risk free rates from June 1964-December 2004 and their RGMM weights using respectively the models of CKLS, Ahn and Gao and Chapman and Pearson.

The weights assigned by the RGMM estimation procedure play an important role in the computation of the IRGMM estimator since they decrease the influence of the outliers 
in the computation of the auxiliary estimator with real data (step 1) in Section 2.2.

Figure 3.3 shows that the RGMM estimation of the CKLS model detects outliers in September 1974, during the period from January 1980 through August 1982, between October-December 1987 and in September 2001. Figure 3.4 shows that in addition to the outliers detected by the RGMM using CKLS model, the RGMM using Ahn and Gao's model detects additional periods with outliers. The most important among them are: December 1968, May 1971, October 1984 and November 1998. Figure 3.5 presents even more outliers detected by the RGMM computed using the model of Chapman and Pearson. These additional outliers are detected in the period between June 2003 and October 2004.

Table 3.3 presents the parameter estimates obtained with the three models and each model is estimated with the three different techniques (t-statistics of the parameter estimates are in parentheses). The IRGMM estimation is performed using $S=100$ pseudo-data sets generated from the fine discretization (11) with $\delta=1 / 22^{5}$ The weighting matrix in (13) is replaced by the inverse of the asymptotic matrix of the RGMM estimator, i.e. $\Omega=E\left[\frac{\partial h_{c}^{A, \tau}}{\partial \mu}\right]^{T} E\left[\frac{\partial h_{c}^{A, \tau}}{\partial \mu}\right]$. We use the Broyden-Fletcher-Goldfarb-Shanno (BFGS) variant of the Davidon-Fletcher-Powell minimization algorithm for which we compute the gradient using Ridders method of polynomial extrapolation. This minimization algorithm consists of finding a local mimimum in the neighborhood of the initial point, for which we choose the parameter estimates obtained with RGMM. Since each evaluation of the function in (13) needs an optimization over $S=100$ simulated datasets, an IRGMM estimation is computationally intensive. All of our programs are executed in $\mathrm{C}$ and are available from the first author.

In order to compare numerically the different estimation techniques we define the following goodness-of-fit measures:

$$
\begin{gathered}
R \widehat{A M S} E=\left(\frac{1}{n} \sum_{t=1}^{n} \frac{1}{S} \sum_{s=1}^{S}\left(y_{t}^{(s)}-y_{t}\right)^{2}\right)^{\frac{1}{2}} \\
\widehat{A M A D}=\frac{1}{n} \sum_{t=1}^{n} \underset{s}{\operatorname{median}}\left(\left|y_{t}^{(s)}-\underset{l}{\operatorname{median}}\left(y_{t}^{(l)}\right)\right|\right), \\
\widehat{A M B}=\frac{1}{n} \sum_{t=1}^{n}\left(\left|\underset{s}{\operatorname{median}}\left(y_{t}^{(s)}\right)-y_{t}\right|\right),
\end{gathered}
$$

where $\left\{y_{t}\right\}_{t=1, \ldots, n}$ are the real observations and $\left\{y_{t}^{(s)}\right\}_{t=1, \ldots, n}^{s=1, \ldots, S}$ are simulated data using parameter estimates and the corresponding model replacing $y_{0}^{s}$ by the real observation $y_{0}$. For instance, for the GMM and the RGMM tests, the $\left\{y_{t}^{s}\right\}$ are generated from the crude discretization (5), and for the IRGMM tests they are generated from (11) with $\delta=1 / 22^{6}$. $\widehat{A M B}$ measures the average median bias, $\widehat{A M A D}$, the variability and $\overparen{R A M S} E$, the root

\footnotetext{
${ }^{5}$ To ensure that the pseudo-observations $y_{k \delta}$ are between 0 and 1 , we set the value of the quadratic function in (13) to $10^{8}$ in the points where the generated pseudo-observations are not all in the interval $] 0,1[$.

${ }^{6}$ For these measures only, $\left(y_{k \delta}\right)^{\gamma}$ in (11) is replaced by $\left|y_{k \delta}\right|^{\gamma}$
} 
mean squared error of the simulated paths. Small $R \widehat{A M S} E$ values show the ability to generate pseudo-data using the parameter estimates similar to the real data series.

In Table 3.3, the goodness-of-fit measures are computed using $S=100$ pseudo-data sets of the same sample size as the real dataset, $n=487$ months. The smallest values of goodness-of-fit measures for each model are underlined. The smallest value of $R \widehat{A M S} E$ for all models and estimation techniques is marked by a star. Table 3.3 shows that for every model the smallest values for $R \widehat{A M S} E$ are obtained using IRGMM parameter estimates, but for different reasons for each model. For the CKLS model, the small $\widehat{A M A D}$ obtained using the IRGMM parameter estimates shows that the gain in the $R \widehat{A M S} E$ is due to the small variability among the simulated paths. For the Ahn and Gao model, the gain using IRGMM is particularly important, especially when compared to the classical GMM. The gain is due in part to the small variability of the simulated paths generated using the IRGMM parameter estimates and also to the fact that the GMM and RGMM parameter estimates for $\beta_{0}$ do not satisfy the condition requiring that the process $\left\{y_{t}^{(s)}\right\}$ stays away from 0 (see Ahn and Gao, 1999, p.731). They can generate data with negative values (though not statistically significant), which, in turn, explain the very large $R \widehat{A M S} E$ values. The IRGMM parameter estimates for Ahn and Gao's model are the only ones satisfying $\beta_{0}>0$. For the model of Chapman and Pearson, the performance of the IRGMM is neither due to smaller variability (which obtains for GMM) nor to a smaller bias (which obtains for RGMM) but a tradeoff between them.

\begin{tabular}{|c|c|c|c|c|c|c|c|c|c|c|c|}
\hline & & $\beta_{0}$ & $\beta_{1}$ & $\beta_{2}$ & $\beta_{3}$ & $\sigma$ & $\gamma$ & $R \widehat{A M S E}$ & $\widehat{A M A D}$ & $\widehat{A M B}$ & $10^{-3}$ \\
\hline \multirow[t]{3}{*}{$C K L S:$} & GMM & $\underset{(1.57)}{0.00172}$ & $\begin{array}{c}-0.03084 \\
(-1.34)\end{array}$ & - & - & $\underset{(1.54)}{0.40320}$ & $\begin{array}{c}1.53091 \\
(6.27)\end{array}$ & 42.96 & 9.08 & $\underline{19.02}$ & \\
\hline & RGMM & $\begin{array}{c}0.00075 \\
(0.94)\end{array}$ & $\begin{array}{c}-0.01031 \\
(-0.59)\end{array}$ & - & - & $\underset{(2.78)}{0.14135}$ & $\underset{(9.06)}{1.17706}$ & 79.92 & 15.75 & 19.36 & \\
\hline & IRGMM & $\begin{array}{c}0.00209 \\
(0.48)\end{array}$ & $\begin{array}{c}-0.04056 \\
(-0.42)\end{array}$ & - & - & $\begin{array}{c}0.20679 \\
(0.28)\end{array}$ & $\begin{array}{c}1.30304 \\
(1.07)\end{array}$ & $\underline{31.80^{*}}$ & $\underline{8.43}$ & 19.72 & \\
\hline \multirow[t]{3}{*}{$A G:$} & GMM & $\begin{array}{c}-0.00163 \\
(-1.44)\end{array}$ & $\underset{(1.76)}{0.08612}$ & $\begin{array}{c}-0.83573 \\
(-1.84)\end{array}$ & - & $\begin{array}{c}0.37165 \\
(20.46)\end{array}$ & 1.5 & 5231.05 & 38.63 & $\underline{20.25}$ & \\
\hline & RGMM & $\begin{array}{c}-0.00001 \\
(-0.01)\end{array}$ & $\begin{array}{c}0.00764 \\
(0.25)\end{array}$ & $\begin{array}{c}-0.05911 \\
(-0.18)\end{array}$ & - & $\begin{array}{c}0.33065 \\
(25.19)\end{array}$ & 1.5 & 69.81 & 16.36 & 20.79 & \\
\hline & IRGMM & $\begin{array}{c}0.00034 \\
(0.21)\end{array}$ & $\begin{array}{c}-0.00993 \\
(-0.18) \\
\end{array}$ & $\begin{array}{c}-0.04187 \\
(-0.06)\end{array}$ & - & $\begin{array}{c}0.33587 \\
(3.72)\end{array}$ & 1.5 & $\underline{39.87}$ & $\underline{5.40}$ & 31.66 & \\
\hline \multirow[t]{3}{*}{$C P:$} & GMM & $\begin{array}{c}-0.00543 \\
(-1.26)\end{array}$ & $\underset{(1.42)}{0.16125}$ & $\begin{array}{c}-1.21467 \\
(-1.54)\end{array}$ & $\begin{array}{c}0.00004 \\
(1.25)\end{array}$ & $\begin{array}{c}0.35025 \\
(1.75)\end{array}$ & $\begin{array}{c}1.47779 \\
(6.90)\end{array}$ & 49.34 & $\underline{1.60}$ & 43.60 & \\
\hline & RGMM & $\begin{array}{c}-0.00185 \\
(-0.53)\end{array}$ & $\begin{array}{c}0.06312 \\
(0.64)\end{array}$ & $\begin{array}{c}-0.50117 \\
(-0.65)\end{array}$ & $\begin{array}{c}0.00001 \\
(0.55)\end{array}$ & $\begin{array}{c}0.11987 \\
(2.57)\end{array}$ & $\begin{array}{c}1.12698 \\
(8.12)\end{array}$ & 43.86 & 19.86 & $\underline{25.81}$ & \\
\hline & IRGMM & $\begin{array}{c}-0.00083 \\
(-0.11)\end{array}$ & $\begin{array}{c}0.04475 \\
(0.52)\end{array}$ & $\begin{array}{c}-0.50026 \\
(-0.16)\end{array}$ & $\underset{(0.08)}{0.00000}$ & $\frac{0.19831}{(0.10)}$ & $\begin{array}{c}1.29488 \\
(0.38)\end{array}$ & $\underline{41.48}$ & 16.07 & 27.38 & \\
\hline
\end{tabular}

Table 3.3: Parameter estimates for monthly Treasury Bill rates from 06/1964-12/2004.

We next consider two subperiods separately: 06/1964-12/1981 and 01/1982-12/2004. This is an useful experiment to investigate the potential for robust GMM estimators since 
most of the outliers detected in Figures 3.3-3.5 are in the first subperiod. The left three columns in Table 3.4 present the goodness-of-fit tests for the first subperiod ${ }^{7}$. Here, the conclusions are similar to those in Table 3.3. For each model, the IRGMM parameter estimates generate the most accurate within-sample predictions of the interest rates. For the CKLS and Chapman and Pearson models, the gain using IRGMM is mainly due to small variability $(\widehat{A M A D})$ of the pseudo-data series. For the Ahn and Gao model the gain is both due to a smaller variability and to the bias correction.

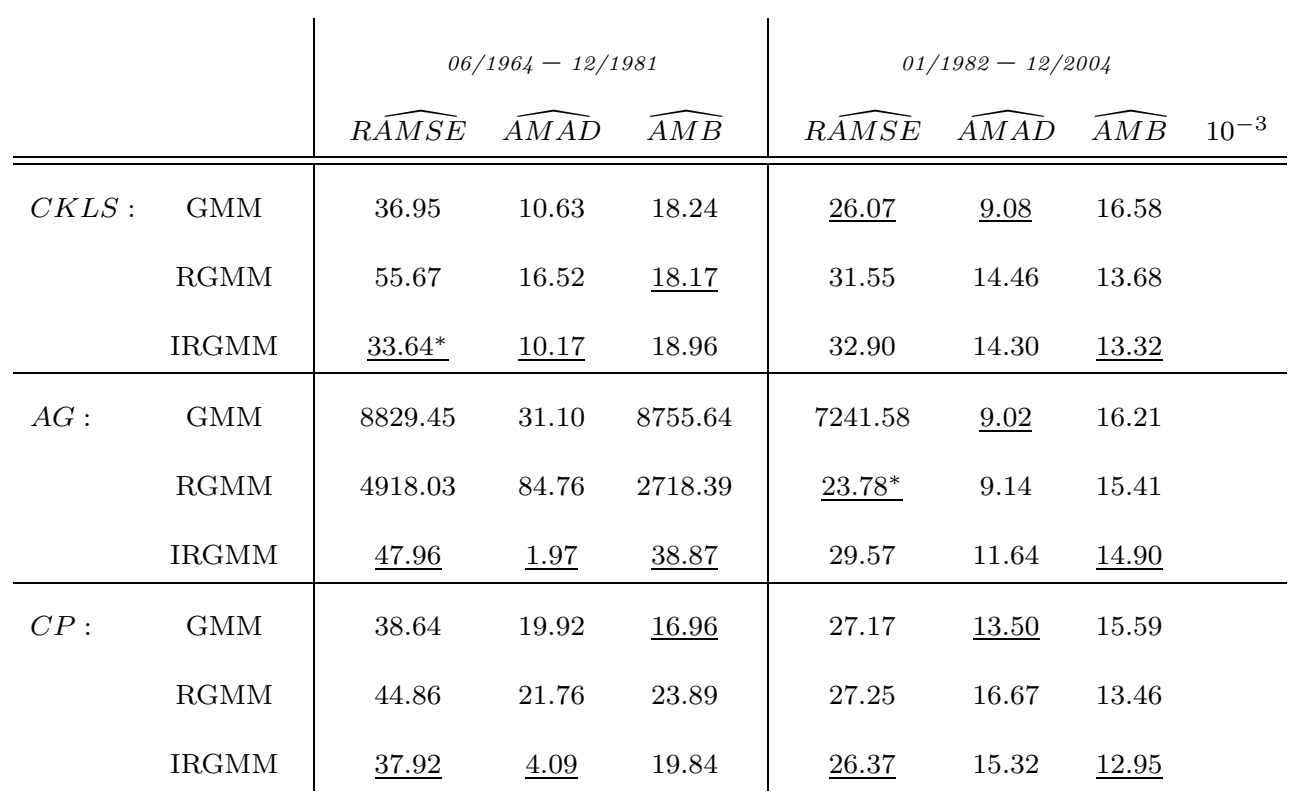

Table 3.4 Parameter estimates for one month risk free rates for the periods 06/1964-12/1981 and 01/1982-12/2004.

The right three columns in Table 3.4 present the goodness-of-fit measures for the subperiod 01/1982-12/2004 where only a few outliers were detected. For the CKLS model, the fact that the GMM has the smallest $R \widehat{A M S} E$ value due to the small variability in the pseudo-data suggests that model misspecification and discretization errors were not important in this case. By constrast, Ahn and Gao's model seems to be more sensitive to outliers since there is an important gain using RGMM relative to GMM. For the Chapman and Pearson model, there is a small gain using IRGMM due to the correction of the discretization bias. In this subperiod, Ahn and Gao's model with RGMM parameter estimates fits best the data overall.

Tables 3.3-3.4 show that Ahn and Gao's model is sensitive to model misspecification error while CKLS and Chapman and Pearson's models are more sensitive to the discretization error. Since the IRGMM technique corrects both errors, the IRGMM parameter estimates often has the smallest $R \widehat{A M S} E$ values.

\footnotetext{
${ }^{7}$ The parameter estimates are available upon request from the authors.
} 


\section{Out-of-Sample forecasts: $2000-2004$}

We consider now monthly US dollar Eurocurrency rates from January 1990 until December 2004. The rates represent 11 a.m. (GMT) quotes in London and are drawn from Datastream International. The time series is plotted in Figure 4.1. We estimate the three models presented in Section 3 with the three different GMM techniques described in Section 2 using ten-year estimation horizons and we assess the out-of-sample performance based on one-year-ahead forecasts (based on twelve monthly observations). We compute forecast accuracy using the same goodness-of-fit measures as in the estimation above, but by replacing $\left\{y_{t}\right\}_{t=1, \ldots, 12}$ by the out-of-sample observations of the eleventh year and by simulating forecasts using the model parameter estimates and replacing $y_{0}^{(s)}$ by the last observation from the ten-year period dataset $y_{n}$, where $n=120$.

Table 4.1 presents the $R \widehat{A M S} E$ goodness-of-fit ${ }^{8}$ values obtained for the one-year-ahead forecasts from five overlapping ten-year estimation horizons: 1990-1999, 1991-2000, 19922001, 1993-2002 and 1994-2003. The smallest values for each model are underlined, and the smallest values across the models are marked by stars. In most cases, the small forecast errors for $R \widehat{A M S} E$ obtain for forecasts based on IRGMM estimated models. There is an important gain by using IRGMM compared to GMM and RGMM, especially in the case of models with non-linear drift. The introduction of the non-linear term $\beta_{3} / y_{t}$ in the drift of Chapman and Pearson's model appears to be important for the forecasts of the years 2003 and 2004.

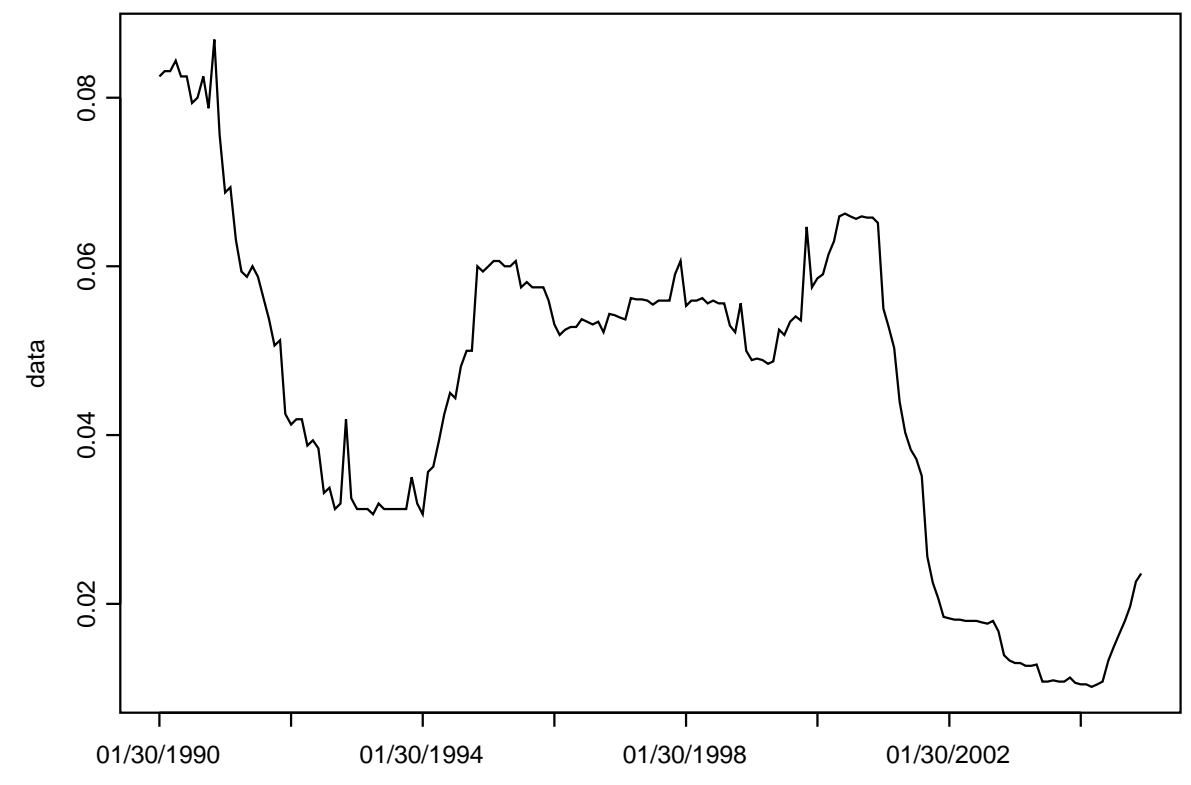

Figure 4.1: Monthly US dollar Eurocurrency rate data from 01/1990 to 12/2004.

\footnotetext{
${ }^{8}$ The results for the other two metrics and the parameter estimates are available from the authors upon request
} 


\begin{tabular}{|c|c|c|c|c|c|c|}
\hline & & \multicolumn{3}{|c|}{$R \widehat{A M S E}$} & $10^{-3}$ & \multirow[b]{2}{*}{2004} \\
\hline & & 2000 & 2001 & 2002 & 2003 & \\
\hline \multirow[t]{3}{*}{$C K L S:$} & GMM & 11.34 & 26.19 & 9.33 & 4.12 & 7.94 \\
\hline & RGMM & 11.22 & 25.94 & 8.12 & 3.64 & 7.88 \\
\hline & IRGMM & $\underline{4.22}^{*}$ & $\underline{24.36}$ & $\underline{1.18}^{*}$ & 3.27 & $\underline{6.63}$ \\
\hline \multirow[t]{3}{*}{$A G:$} & GMM & $\underline{10.37}$ & 23.90 & 559.95 & 4.99 & 9.89 \\
\hline & RGMM & 10.55 & $\underline{23.87}^{*}$ & 107.42 & $\underline{2.35}$ & 14.30 \\
\hline & IRGMM & 11.86 & 24.01 & $\underline{5.41}$ & 2.73 & $\underline{8.65}$ \\
\hline \multirow[t]{3}{*}{$C P:$} & GMM & 11.13 & 24.77 & 615.24 & 4.43 & $\underline{4.97}^{*}$ \\
\hline & RGMM & 11.16 & 25.21 & 186.86 & 2.73 & 5.16 \\
\hline & IRGMM & $\underline{9.41}$ & 24.69 & $\underline{43.72}$ & $2.19 *$ & 5.22 \\
\hline
\end{tabular}

Table 4.1 Goodness-of-fit tests of the forecast of US dollar Eurocurrency rates for the years 2000-2004.

Next, we turn to out-of-sample forecasts of the monthly Eurocurrency rates for the UK and Switzerland. These data are also based on 11 a.m. (GMT) quotes in London and are drawn from Datastream International.

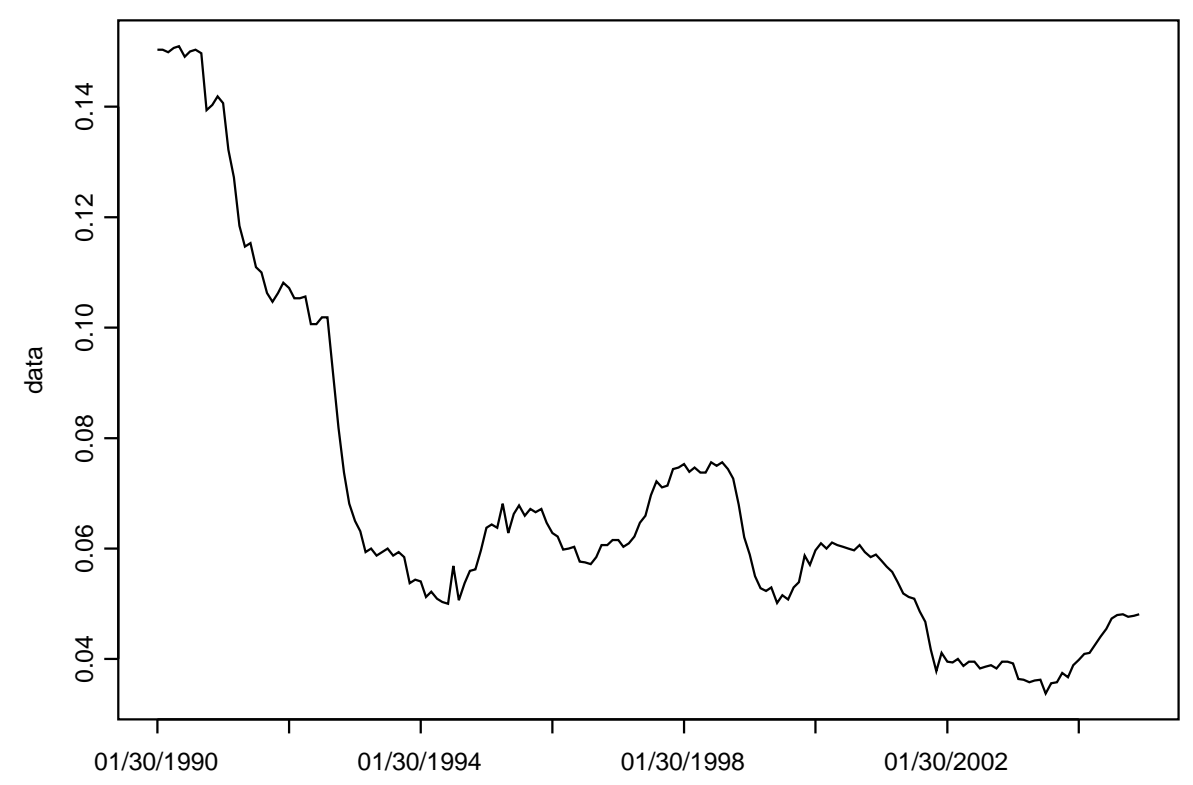

Figure 4.2: Monthly UK Sterling Eurocurrency rate data from 01/1990 to 12/2004. 


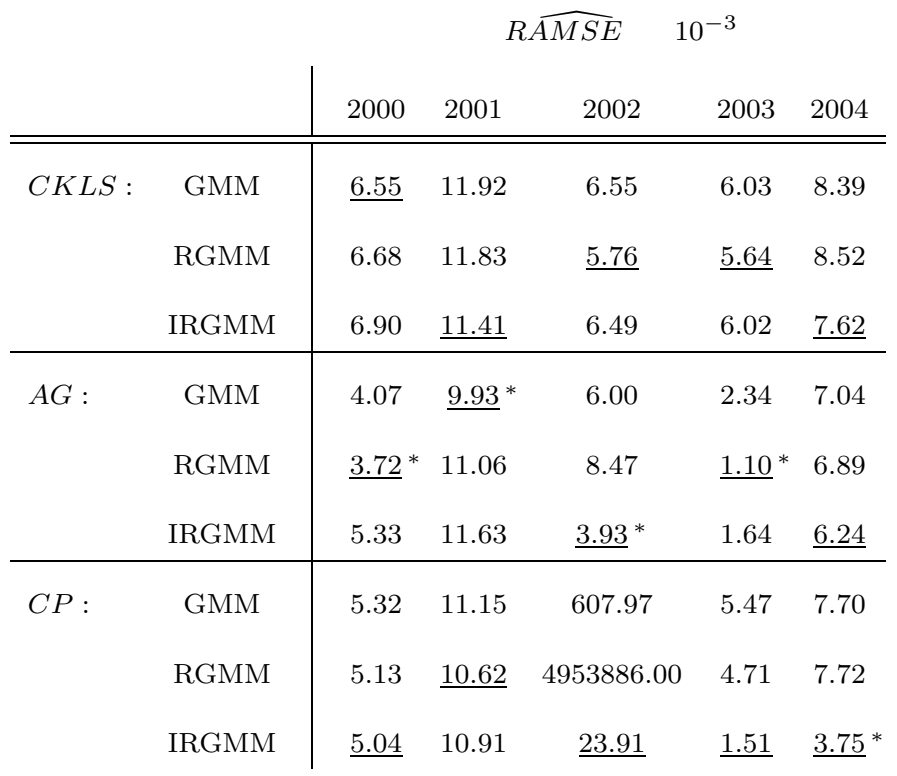

Table 4.2 Goodness-of-fit tests of the forecasts of UK Sterling Eurocurrency rates for the years $2000-2004$.

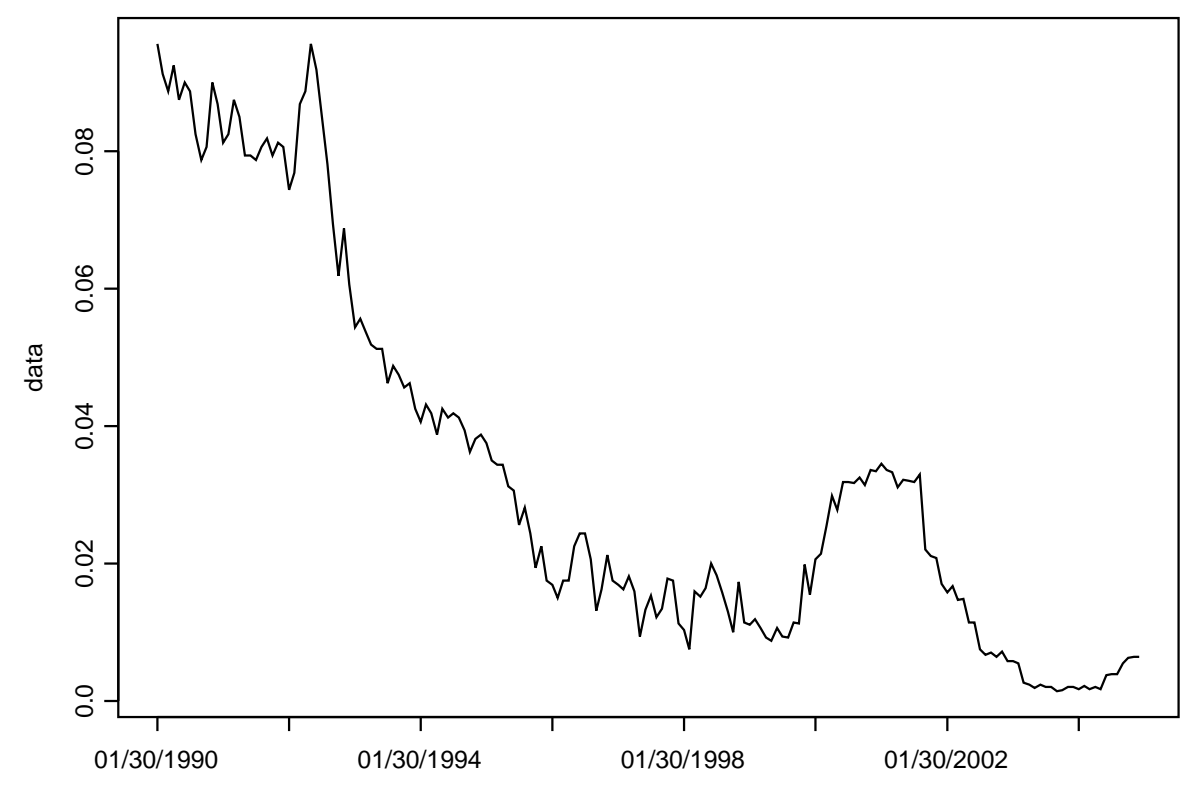

Figure 4.3: Monthly Swiss Franc Eurocurrency rate data from 01/1990 to 12/2004. 


\begin{tabular}{cc|ccccc} 
& & \multicolumn{6}{c}{$R \widehat{A M S E}$} & $10^{-3}$ \\
\hline \hline \multirow{2}{*}{ CKLS: } & GMM & 15.96 & 9.48 & 9.55 & 10.73 & $\underline{6.85}$ \\
& RGMM & 15.93 & $\underline{9.20}$ & 9.89 & 11.25 & 7.38 \\
& IRGMM & $\underline{12.61}{ }^{*}$ & 10.64 & $\underline{8.79}$ & $\underline{4.13}$ & 11.89 \\
\hline \multirow{2}{*}{$A G:$} & GMM & 16.76 & $\underline{4.47}$ & 6.36 & 4.85 & 4.75 \\
& RGMM & 17.71 & 4.47 & 5.36 & $\underline{1.11}$ & 3.90 \\
& IRGMM & $\underline{16.08}$ & 4.70 & $\underline{5.23}$ & 1.17 & $\underline{2.68}$ \\
\hline \multirow{2}{*}{$C P:$} & GMM & 15.40 & 8.60 & $\underline{7.66}$ & 38.50 & 1242.80 \\
& RGMM & 15.44 & $\underline{8.40}$ & 8.02 & 18.20 & $\underline{11.51}$ \\
& IRGMM & $\underline{15.24}$ & 10.35 & 9.18 & $\underline{12.68}$ & 18.03
\end{tabular}

Table 4.3 Goodness-of-fit tests of the forecasts of Swiss Franc Eurocurrency rates for the years 2000-2004.

Figures 4.2 and 4.3 plot the Eurocurrency rates for the UK Sterling and Swiss Franc from January 1990 until December 2004. Tables 4.2 and 4.3 present the out-of-sample forecasts of the years 2000-2004 for the UK Sterling and Swiss Franc. For both countries, the comparison across models shows that the model of Ahn and Gao best fits the data. In most of the cases, the IRGMM yielded the more accurate forecasts, followed by RGMM and occasionally GMM. In the few cases when the GMM forecasts performed best, the gain compared to the other estimation techniques is not significant. On the other hand, the gain using IRGMM and sometimes RGMM compared to GMM can be substantial, especially in the models with nonlinear drift. Consider, for instance, the years 2003 and 2004 for AG and $\mathrm{CP}$ for the Swiss Franc rates. Finally, the gain using IRGMM can be even more important when compared to RGMM. Consider, for instance, the year 2002 for UK Sterling rates.

\section{Conclusion}

In this paper we have proposed an application of the indirect robust GMM (IRGMM) simulation-based estimation technique for models of short-term interest rates. We presented an empirical comparison of three estimation techniques (GMM, RGMM and IRGMM) for three different interest-rate diffusion models that have received attention in the finance literature. We evaluated the within-sample performance of these techniques for US interest rates between 1964 and 2004. We then compared the out-of-sample predictive power of the three techniques on monthly Eurocurrency rates for the US dollar, UK Sterling and Swiss Franc. We find that the improvement in goodness-of-fit provided by IRGMM estimation 
tends to occur most consistently for those models with more complex specifications, such as those with non-linear drift.

A possible future research direction would be to go beyond single factor models and to investigate the performance of IRGMM in more complex diffusion models.

\section{References}

Ahn, D.H. and Gao, B. (1999), "A parametric nonlinear model of term structure dynamics", The Review of Financial Studies, 12, 721-762.

Aït-Sahalia, Y. (1996), "Testing Continuous-Time Models of the Spot Interest Rate", Review of Financial Studies, 9, 385-426.

Aït-Sahalia, Y. (1999), "Transition Densities for Interest Rate and Other Nonlinear Diffusions", Journal of Finance, 54, 1361-1395.

Aït-Sahalia, Y. (2002), "Maximum Likelihood Estimation of Discretetely Sampled Diffusions: a Closed-form Approximation Approach", Econometrica, 70, 223-262.

Andersen, T.G and Lund, J. (1997), "Estimating Continuous Time Stochastic Volatility Models of the Short Term Interest Rate", Journal of Econometrics, 77, 343-377.

Beskos, A., Papaspiliopoulos, O., Roberts, G. O. and Fearnhead, P. (2006), "Exact and computationally efficient likelihood-based estimation for discretely observed diffusion processes", Journal of the Royal Statistical Society, 68, 1-29.

Brennan, M. J. and Schwartz, E. S. (1977), "Savings Bonds, Retractable Bonds, and Callable Bonds", Journal of Financial Economics, 3, 133-155.

Brenner, R.J., Harjes, R.H. and Kroner, K.F. (1996), "Another look at models of the short-term interest rate", Journal of Financial and Quantitative Analysis, 31, 85-107.

Broze, L., Scaillet, O. and Zakoïan, J.-M. (1995), "Testing for Continuous-time Models of the Short-term Interest Rate", Journal of Empirical Finance, 2, 199-223.

Chan, K. C., Karolyi, G. A., Longstaff, F. A. and Sanders, A. B. (1992), "An Empirical Comparison of Alternative Models of the Short-Term Interest Rate", The Journal of Finance, 47, 1209-1227.

Chapman, D., Long, J. and Pearson, N.D. (1999), "Using Proxies for the Short-Rate: When are Three Months Like an Instant?", Review of Financial Studies, 12, 763-806.

Chapman, D.A. and Pearson, N. D. (2000), "Is the Short Rate Drift Actually Nonlinear?", Journal of Finance, 55, 355-388.

Conley, T.G., Hansen, L.P., Luttmer, E.G.J. and Scheinkman, J.A. (1997), "Short-Term 
Interest Rates as Subordinated Diffusions", Review of Financial Studies, 10, 525-578.

Cox, J.C., Ingersoll, J.E. and Ross, S.A. (1981), "A re-examination of traditional hypotheses about the term structure of interest rates," Journal of Finance, 36, 769-799.

Cox, J.C., Ingersoll, J.E. and Ross, S.A. (1985), "A Theory of the Term Structure of Interest Rates", Econometrica, 53, 385-407.

Dell'Aquila, R., Ronchetti, E. and Trojani, F. (2003), "Robust GMM Analysis of Models for the Short Rate Process", Journal of Empirical Finance, 10, 373-397.

Dothan, U.L. (1978), "On the Term Structure of Interest Rates", Journal of Financial Economics, 6, 59-69.

Durham, G.B. (2003), "Likelihood-based specification analysis of continuous-time models of the short-term interest rate", Journal of Financial Economics, 70, 463-487.

Durham, G.B., and Gallant, A.R. (2002), "Numerical Techniques for Maximum Likelihood Estimation of Continuous-Time Diffusion Processes", Journal of Business and Economic Statistics, 20, 279-316.

Elerian, O., Chib, S. and Shephard, N. (2001), "Likelihood Inference for Discretely Observed Nonlinear Diffusions", Econometrica, 69, 959-994.

Eraker, B. (2001), "MCMC Analysis of Diffusion Models with Application to Finance", Journal of Business and Economic Statistics, 19, 177-191.

Gallant, R. and Tauchen, G. (1996), "Which Moments to Match?", Econometric Theory, 12, 657-681.

Genton, M. G. and Ronchetti, E. (2003), "Robust Indirect Inference", Journal of the American Statistical Association, 98, 67-76.

Gouriéroux, C. and Monfort, A. (1996), Simulation-Based Econometric Methods, Oxford University Press.

Gouriéroux, C., Monfort, A. and Renault, E. (1993), "Indirect Inference", Journal of Applied Econometrics, 8, S85-S118.

Hong, Y. and Li, H. (2005), "Nonparametric Specification Testing for Continuous-Time Models with Applications to Term Structure of Interest Rates", The Review of Financial Studies, 18, 37-84.

Johannes, M. (2004), "The Statistical and Economic Role of Jumps in Interest Rates", Journal of Finance, 59, 227-260.

Kalimipalli, M. and Susmel, R. (2004), "Regime-switching Stochastic Volatility and Shortterm Interest Rates", Journal of Empirical Finance, 11, 309-329. 
Ortelli, C. and Trojani, F. (2005), "Robust Efficient Method of Moments", Journal of Econometrics, 128, 69-97.

Pritsker, M. (1998), "Nonparametric Density Estimation and Tests of Continuous Time Interest Rate Models", Review of Financial Studies, 11, 449-87.

Ronchetti, E. and Trojani, F. (2001), "Robust Inference with GMM Estimators", Journal of Econometrics, 101, 37-69.

Stanton, R. (1997), "A Nonparametric Model of Term Structure Dynamics and the Market Price of Interest Rate Risk", Journal of Finance, 52, 1973-2002.

Vasicek, O. (1977), "An Equilibrium Characterization of the term structure.", Journal of Financial Economics, 5, 177-188. 